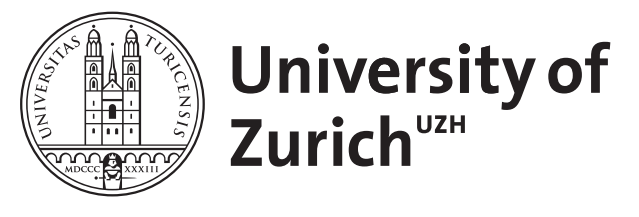

\title{
Ösophagotracheale Fistel
}

Böttcher-Haberzeth, S ; Weber, D ; Meuli, M ; Sacher, P

\begin{abstract}
Verschluckte, im Ösophagus stecken gebliebene, Knopfbatterien müssen notfallmäßig entfernt werden, da sie trotz rascher Entfernung in kürzester Zeit durch verschiedene Mechanismen zu tiefen Verletzungen der Mukosa führen können. Dies wird anhand eines Fallbeispiels eines 2-jährigen Mädchens demonstriert, das nach rascher, unproblematischer endoskopischer Entfernung einer Knopfbatterie aus dem Ösophagus und einem zunächst guten Verlauf eine ösophagotracheale Fistel entwickelte
\end{abstract}

DOI: https://doi.org/10.1007/s10049-005-0753-3

Posted at the Zurich Open Repository and Archive, University of Zurich

ZORA URL: https://doi.org/10.5167/uzh-156098

Journal Article

Published Version

Originally published at:

Böttcher-Haberzeth, S; Weber, D; Meuli, M; Sacher, P (2005). Ösophagotracheale Fistel. Notfall Rettungsmedizin, 8(5):342-345.

DOI: https://doi.org/10.1007/s10049-005-0753-3 
Notfall + Rettungsmedizin $2005 \cdot 8: 342-345$

DOI 10.1007/s10049-005-0753-3

Online publiziert: 28. Juli 2005

(c) Springer Medizin Verlag 2005

\section{Redaktion}

B. Gorgaß, Solingen

T. Pohlemann, Hannover

A. Thierbach, Mainz

S. Böttcher-Haberzeth · D. Weber · M. Meuli · P. Sacher · Kinderspital Zürich

\section{O̊sophagotracheale Fist
Hervorgerufen durch eine im Ösophagus stecken gebliebene Knopfbatterie}

und ein weiches Abdomen ohne Druckdolenzen.

\section{Diagnostik}

Eine Röntgenaufnahme des Thorax a.p. stellte einen kreisrunden Fremdkörper auf Höhe der Aortenenge dar. Seine frontale und aufrechte Lage bestätigte die Lokalisation im Ösophagus, die deutlich sichtbare typische Doppelkontur deutete auf eine Knopfbatterie hin (• Abb. 1). Ansonsten normal belüftete Lungen ohne Hinweis für eine Aspiration.

\section{Therapie und Verlauf}

\section{Fallbericht}

\section{Anamnese}

Ein 2-jähriges Mädchen wird von seinen Eltern auf die Notfallstation gebracht, weil der Vater selbst kurz zuvor die Ingestion eines Fremdkörpers beobachtet hatte. Er berichtete, dass es sich höchstwahrscheinlich um eine Knopfbatterie handle, da eine solche Batterie in seinem Taschenrechner fehle und diese kurz zuvor noch vorhanden gewesen war. Dem Mädchen ging es zunehmend schlechter, sie hustete, produzierte vermehrt Schleim und verweigerte jegliche Nahrungs- und Flüssigkeitsaufnahme.

\section{Klinischer Befund}

Das Mädchen präsentierte sich in einem deutlich reduzierten Allgemeinzustand, zeigte aber eine ruhige Atmung mit normalem pulmonalen Auskultationsbefund

\section{Maßnahmen bei Erstaufnahme}

Nach Diagnosestellung wurde die Batterie sofort endoskopisch entfernt, was problemlos möglich war. Die Mukosa zeigte nach der Entfernung eine deutliche dunkle Verfärbung der Vorderwand (• Abb. 2) mit einer oberflächlichen Verletzung, jedoch ohne Hinweise auf eine Perforation. Auch die Batterie selbst wies deutliche Oberflächenveränderungen auf $(\bullet A b b .3 a, b)$. Das zeitliche Intervall zwischen Ingestion und Entfernung der Batterie betrug $2 \mathrm{~h}$.

Im Wissen um die Gefahren, die eine Knopfbatterie bringen kann, wurde das Mädchen stationär aufgenommen. Sie erhielt eine prophylaktische Antibiotikatherapie mit Metronidazol, Gentamicin und Amoxicillin, und die Ernährung wurde über eine in der Narkose eingelegten Magensonde verabreicht. Vor Beginn der peroralen Nahrungsaufnahme, 3 Tage nach der Fremdkörperentfernung, wurde ein Ösophagogramm durchgeführt, das un- auffällige Befunde zeigte, insbesondere ohne Hinweis auf eine Stenose oder Perforation. Der Kostaufbau verlief problemlos, und die Patientin konnte 3 Tage später nach Hause entlassen werden.

\section{Wiederaufnahme}

Zwei Tage nach der Entlassung, d. h. 8 Tage nach Ingestion der Batterie, präsentierte sich das Mädchen erneut auf unserer Notfallstation wegen einer deutlichen Verschlechterung des Allgemeinzustandes, starkem Husten und einer vermehrten Salivation. Eine Röntgenaufnahme des Thorax wurde als normal befundet. Ein erneutes Ösophagogramm (• Abb. 4) stellte nun eine ösophagotracheale Fistel im oberen Drittel des Ösophagus dar, $13 \mathrm{~cm}$ ab Zahnreihe, mit Übertritt des Kontrastmittels in das Bronchialsystem, sodass die Fistel operativ verschlossen werden musste. Die vorgängige Tracheoskopie zeigte die Perforationsstelle ca. $4 \mathrm{~cm}$ unterhalb des Cricoids mit einer Länge von ca. $1 \mathrm{~cm}$. In der Ösophagoskopie bestätigte sich eine Perforationsstelle ebenfalls mit einem Durchmesser von ca. $1 \mathrm{~cm}$, der übrige Ösophagus war unauffällig. Ein paramedianer, zervikaler Zugang rechts wurde gewählt. Es zeigte sich eine entzündlich veränderte Perforationsstelle sowohl der anterioren Wand des Ösophagus wie auch der posterioren Wand der Trachea mit einem Durchmesser von ca. $1 \mathrm{~cm}$. Das nekrotische Gewebe wurde entfernt und sowohl die Ösophaguswand wie auch die Pars membranacea der Trachea primär verschlossen. Die histologische Untersuchung des Resektates ergab „Ösophaguswandfragmente mit 
tief reichender Ulzeration und chronisch granulierender Entzündung sowie reichlich Nachweis von Eisenpigment“.

Der postoperative Verlauf war problemlos. Die Patientin konnte noch am gleichen Tag extubiert werden. Nach 4 Tagen mit parenteraler Ernährung erfolgte der Nahrungsaufbau via Magensonde. Nach einem unauffälligen Ösophagogramm, 8 Tage postoperativ, verlief der Übergang zur peroralen Nahrungsaufnahme ebenfalls ohne weitere Probleme. Das Mädchen konnte 2 Wochen nach operativem Verschluss der Fistel nach Hause entlassen werden. Weitere Ösophagogramme nach 1 Woche sowie nach 1 Monat zeigten keine pathologischen Befunde.

\section{Diskussion}

Knopfbatterien stellen eine große Gefahr dar, wenn sie im Ösophagus stecken bleiben. Über verschiedene Mechanismen kann die Schleimhaut in kürzester Zeit verletzt werden [3], und es kann zu tiefen Ulzerationen und Perforationen kommen, wobei die elektrochemische Verbrennung die schwerwiegendsten Verletzungen verursacht [9].

\section{(2) Die elektrochemische Verbrennung verursacht die schwerwiegendsten Verletzungen}

Folgende 4 Mechanismen kommen für die Gewebszerstörung in Frage:

1. Elektrochemische Verbrennung: Die Knopfbatterie besteht aus einer Anode und einer Kathode, die durch einen Isolationsring voneinander getrennt sind. Sobald sich ein elektrischer Stromkreis entwickelt, beginnt eine Elektrolyse, und es kommt zu Veränderungen der Schleimhaut. An der an die Kathode angrenzenden $\mathrm{Mu}$ kosa kommt es zu schwerwiegenden Veränderungen mit Verfärbungen, Ulzerationen und tief greifenden Verletzungen. An der Mukosa, die der Anode anliegt, kommt es vor allem zu Ödemen $[5,9]$.

2. Verätzung durch korrosive Substanzen: Durch die Verletzung der Oberfläche der Batterie können korrosive Substanzen freigesetzt werden, welche die
Schleimhaut noch zusätzlich schädigen [1].

3. Toxische Substanzen:

Die Batterie kann toxische Substanzen, wie z. B. Quecksilber, Lithium oder Zink, abgeben, die dann in der Folge zu Toxizitäten führen können $[4,10]$.

4. Drucknekrose

Das Ausmaß der Verletzung hängt ebenfalls von verschiedenen Faktoren ab:

1. Ausrichtung der Batterie:

An der zur Kathode anliegenden Mukosa kommt es zu tieferen Verletzungen $[5,9]$.

2. Größe der Knopfbatterie:

Erst ab einer gewissen Größe kann die Batterie im Ösophagus stecken bleiben und Verletzungen verursachen. In der Literatur werden vor allem Knopfbatterien mit einem Durchmesser von über $15 \mathrm{~mm}$ als problematisch beschrieben, da sie am ehesten im Ösophagus stecken bleiben können $[3,4,10]$. In unserem Fall zeigte die Knopfbatterie einen Durchmesser von $20 \mathrm{~mm}$.

3. Verweildauer:

Je länger, desto größer ist das Ausmaß der Verletzung $[9,10]$.

Eine Gewebszerstörung findet statt, sobald eine Knopfbatterie im Ösophagus stecken bleibt und sich ein elektrischer Stromkreis bilden kann. Ist hingegen eine Batterie leer, kann keine Elektrolyse mehr stattfinden, und dieser Verletzungsmechanismus tritt nicht ein [4].

Im vorgestellten Fall bleibt unklar, ob die ösophagotracheale Fistel z. Z. des ersten Ösophagogramms noch nicht bestanden hat oder ob sie nicht dargestellt werden konnte. Auf alle Fälle muss bei einer verschluckten, im Ösophagus stecken gebliebenen Knopfbatterie zu jedem Zeitpunkt des Verlaufs an eine Perforation oder Fistel gedacht werden.

\section{Bei einer im Ösophagus stecken gebliebenen Knopf- batterie muss immer an eine Perforation oder Fistel gedacht werden}

Wenn eine verschluckte Knopfbatterie den Magen erreicht hat, passiert sie den restli-
Notfall + Rettungsmedizin 2005 $\cdot 8: 342-345$ DOI 10.1007/s10049-005-0753-3

○) Springer Medizin Verlag 2005

S. Böttcher-Haberzeth · D. Weber

M. Meuli $\cdot$ P. Sacher

Ösophagotracheale Fistel. Hervorgerufen durch eine im Ösophagus stecken gebliebene Knopfbatterie

\section{Zusammenfassung}

Verschluckte, im Ösophagus stecken gebliebene, Knopfbatterien müssen notfallmäßig entfernt werden, da sie trotz rascher Entfernung in kürzester Zeit durch verschiedene Mechanismen zu tiefen Verletzungen der Mukosa führen können. Dies wird anhand eines Fallbeispiels eines 2-jährigen Mädchens demonstriert, das nach rascher, unproblematischer endoskopischer Entfernung einer Knopfbatterie aus dem Ösophagus und einem zunächst guten Verlauf eine ösophagotracheale Fistel entwickelte.

\section{Schlüsselwörter}

Knopfbatterie - Ösophagus .

Ösophagotracheale Fistel · Fremdkörper · Ingestion

An esophagotracheal fistula. Developed after impaction of a button battery in the esophagus

\section{Abstract}

Button batteries which are lodged in the esophagus can rapidly lead to deep injuries of the mucosa. Even prompt removal does not preclude the development of an esophagotracheal fistula. Therefore, these objects must be removed immediately. The lesions are a result of different mechanisms. Our report discusses a 2-year-old child who developed an esophagotracheal fistula several days after the unproblematic, rapid endoscopic removal of a button battery lodged in the esophagus.

Keywords

Button battery - Esophagus .

Esophagotracheal fistula · Foreign body . Ingestion 


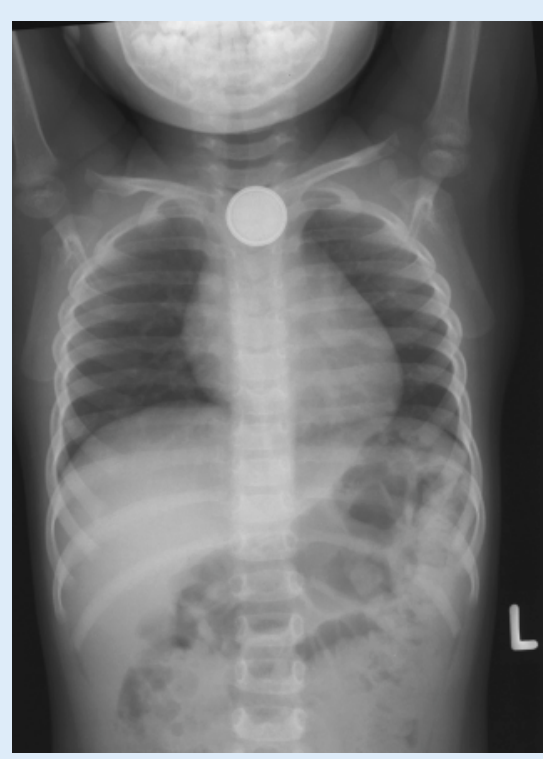

Abb. $1 \Delta$ Röntgen-Thorax a.p. mit Fremdkörper: Knopfbatterie im Ösophagus auf Höhe der Aortenenge mit typischer Doppelkontur

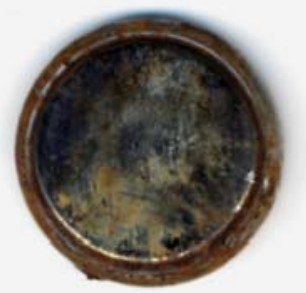

a

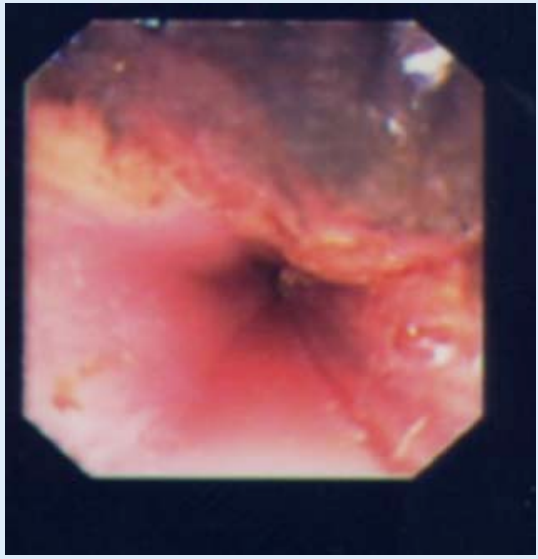

Abb. $2 \Delta$ Ösophagusmukosa mit deutlicher Verfärbung nach Entfernung der Knopfbatterie

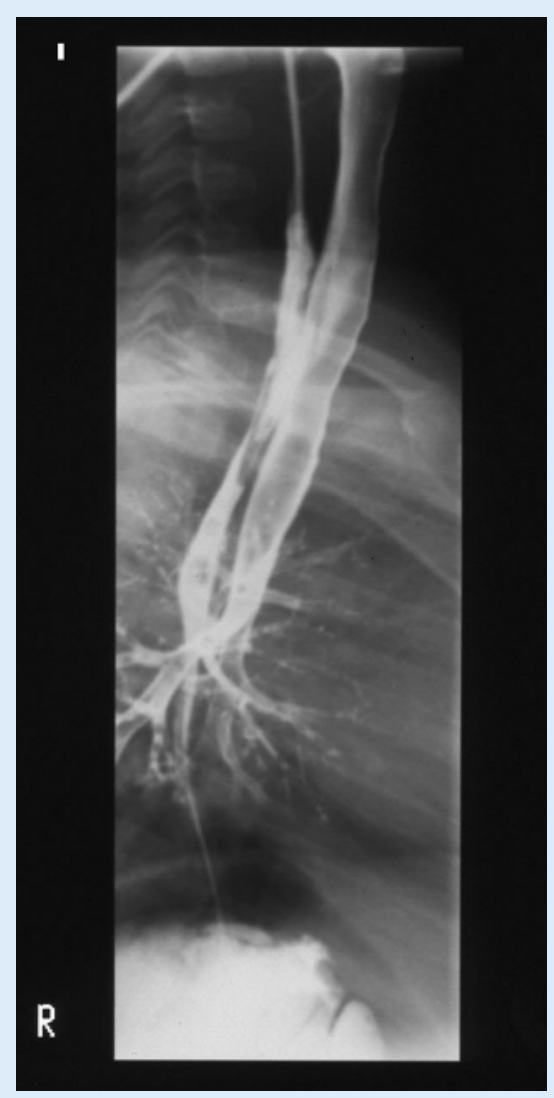

Abb. 4 Ösophagogramm mit sichtbarer ösophagotrachealer Fistel
Abb. 3a,b $<$ Entfernte Knopfbatterie zeigt deutliche Oberflächenveränderung auf der Vorder- und Rückseite chen Gastrointestinaltrakt meistens spontan und wird in der Regel ohne weitere Probleme ausgeschieden [6].

Falls eine Knopfbatterie jedoch kaputt ist oder für längere Zeit im Magen verbleibt, können vermehrt toxische und korrosive Substanzen aus der Batterie austreten, die Mukosaverletzungen und Intoxikationen verursachen $[4,7]$. Bei Batterien im Dünndarm sind in der Literatur, abgesehen von äußerst seltenen Komplikationen, wie z. B. die Perforation eines Meckel-Divertikels, keine Komplikationen beschrieben [2, 6]. Wie man in diesen Fällen jedoch vorgeht, ob und wann eine Entfernung der Knopfbatterie durchgeführt werden soll, darüber ist man sich in der Literatur nicht ganz einig $[1,7]$.

\section{Empfohlene Vorgehensweise}

Die Mehrzahl der Autoren schlägt zurzeit das folgende Vorgehen vor:

Um die Propulsion der Batterie aus dem Magen zu dokumentieren, sollen täglich Abdomenleerbilder angefertigt werden $[2,4,8,9]$. Falls die Knopfbatterie nicht innerhalb von $24 \mathrm{~h}$ in den Dünndarm befördert wird, klinische Symptome einer Mukosaverletzung auftreten, erhöhte Quecksilberblutwerte gemessen werden oder die Batterie radiologische Zeichen einer Leckage aufweist, wird eine endoskopische oder chirurgische Entfernung empfohlen $[2,4,6]$.

Da die saure Umgebung des Magens die Korrosion der Oberfläche der Batterie fördert und somit die Freisetzung von korrosiven und toxischen Substanzen erleich- tert wird, empfiehlt man bei einer intragastrischen Lokalisation die Gabe von $\mathrm{H}_{2}$ Rezeptorenblockern [2, 4].

Quecksilberhaltige Batterien sind aufgrund ihrer weiten Verbreitung und ihrem hohen Inhalt an Quecksilber die gefährlichsten Batterien in Bezug auf mögliche Vergiftungen. Die in der Literatur beschriebenen Intoxikationen bei Kindern sind sehr selten und beschränken sich vor allem auf Fälle von Quecksilber [8]. Der Blutspiegel sollte daher bei quecksilberhaltigen Batterien regelmäßig überprüft werden und ggf. eine Therapie mit einem Chelat-.bildenden Medikament eingesetzt werden $[2,4]$.

Sobald die Batterie den Dünndarm erreicht, wird eine konservative Haltung mit klinischer Beobachtung empfohlen. Ein chirurgischer Eingriff ist nur dann nötig, 
wenn klinische Symptome oder Zeichen einer intestinalen Läsion auftreten [4, 9].

Läsionen kommen also vor allem in Situationen vor, in denen die Batterie längere Zeit in derselben anatomischen Struktur stecken bleibt.

\section{Fazit für die Praxis}

Bei einer verschluckten und im Ösophagus stecken gebliebenen Knopfbatterie handelt es sich um einen Notfall, da sich in sehr kurzer Zeit schwerwiegende Verletzungen des Ösophagus ergeben können. Da bei einem Verdacht auf Fremdkörperingestion oder -aspiration häufig der Rettungsdienst alarmiert wird, sollten sowohl der Notarzt als auch das nichtärztliche Rettungsdienstpersonal über die möglichen Komplikationen dieser Situation informiert sein. Auch bei zunächst weniger eindrucksvoller Symptomatik ist generell eine Klinikeinweisung notwendig. Ein Verdacht auf Ingestion einer Knopfbatterie muss mit höchster zeitlicher Dringlichkeit behandelt werden, um einer möglichen Schädigung der Schleimhaut zuvorzukommen.

Nach Diagnosestellung muss die Batterie so schnell wie möglich endoskopisch entfernt werden. Nach Entfernung der Knopfbatterie sollte die Ösophagoskopie zur Kontrolle der Mukosa wiederholt werden. Anschließend empfehlen wir die Durchführung einer Tracheoskopie, um Veränderungen der Trachea zu erfassen. Da die Gefahr einer ösophagotrachealen Fistel trotz rascher Entfernung besteht, muss auch nach anfänglich günstigem Verlauf und bei unauffälligem Ösophagogramm an eine solche gedacht und aktiv danach gesucht werden.

\section{Korrespondierender Autor \\ Dr. S. Böttcher-Haberzeth}

Kinderspital, Steinwiesstrasse 75, 8032 Zürich, Schweiz

E-Mail: Sophie.Boettcher@kispi.unizh.ch

Interessenkonflikt: Der korrespondierende Autor versichert, dass keine Verbindungen mit einer Firma, deren Produkt in dem Artikel genannt ist, oder einer Firma, die ein Konkurrenzprodukt vertreibt, bestehen.

\section{Literatur}

1. Arana A, Hauser B, Hachimi-Idrissi S, Vandenplas $Y$ (2001) Management of ingested foreign bodies in childhood and review of the literature. Eur J Pediatr 160:468-472

2. El-Barghouty N (1991) Management of disc battery ingestion in children. $\mathrm{Br} J \mathrm{Surg}$ 78:247

3. Imamoglu M, Cay A, Kosucu P et al. (2004) Acquired tracheo-esophageal fistulas caused by button battery lodged in the esophagus. Pediatr Surg Int 20:292-294

4. Laugel V, Beladdale J, Escande B, Simeoni U (1999) Lingestion accidentelle de pile-bouton. Arch Pédiatr 6:1231-1235

5. Lin VYW, Daniel SJ, Papsin BC (2004) Button batteries in the ear, nose and upper aerodigestive tract. International J Pediatr Otorhinolaryngol 68:473479

6. Mallon PT, White JS, Thompson RLE (2004) Systemic absorption of lithium following ingestion of a lithium button battery. Human Experimental Toxicol 23:193-195

7. Namasivayam S (1999) Button battery ingestion: a solution to a management dilemma. Pediatr Surg Int 15:383-384

8. Rebhandl W, Steffan I, Schramel P et al. (2002) Release of toxic metals from button batteries retained in the stomach: an in vitro study. J Pediatr Surg 37(1):87-92

9. Tibbals J, Wall R, Velandy Kootayi S et al. (2002) Tracheo-oesophageal fistula caused by electrolysis of a button battery impacted in the oesophagus. $\mathrm{Ja}$ ediatr Child Health 38:201-203

10. Yardeni D, Yardeni H, Coran AG, Golladay ES (2004) Severe esophageal damage due to button battery ingestion: can it be prevented? Pediatr Surg Int 20:496-501

\section{Jahre Leitende Notarzt- gruppe Hamburg}

In diesen Tagen kann die Leitende Notarztgruppe Hamburg auf ihr 20-jähriges Bestehen zurückblicken.

Unter dem Eindruck eines Unglücks auf dem Hamburger Dom mit mehreren Toten und Schwerverletzten wurde am 1. September 1985 das damals einmalige Konzept zur notfallmedizinischen Versorgung bei Großunfällen durch die Indienststellung der Leitenden Notarztgruppe (LNG) Hamburg von der Behörde für Arbeit, Gesundheit und Soziales umgesetzt.

Um den hohen Standard der Individualmedizin auch bei Anfall einer größeren Patientenzahl sicherzustellen, bedarf es - neben materiellen und personellen Ressourcen besonderer Organisationsformen. So wurde die LNG 1999 in die Feuerwehr Hamburg als Träger des Rettungsdienstes integriert und maßgeblich in die Strukturierung des Einsatzgeschehens eingebunden. Dafür wurde dem LNA 2001 der Organisatorische Leiter Rettungsdienst als Führungsassistent zur Seite gestellt. In Kooperation mit Feuerwehr und Hilfsorganisationen werden die Konzepte zur Bewältigung eines Großschadensereignisses ständig überprüft und verbessert. So ist durch die 1998 eingeführte Patientenanhängekarte eine rasche Registrierung auch größerer Patientengruppen möglich. Die zeitgleiche Beschaffung von Zelten und Neuorganisation der Großunfallsets verschaffte auch die materiellen Voraussetzungen für die Bewältigung des Massenanfalls von Verletzten. Die daraus folgende Neukonzeption der personellen Ansprüche führten u. a. zum Konzept der Spezialeinsatzgruppe (SEG) Rettungsdienst, die eine dringend benötigte Personalreserve für den LNA darstellt.

Die Memplex-Datenbank zur Einschätzung der Toxizität von Giftstoffen, die neuen Infektionsrettungswagen der Feuerwehr zur raschen Eindämmung von Infektionsherden und die Beteiligung an Havariekommandos für die deutschen Hoheitsgewässer sind nur einige Beispiele für die umfassenden Neuerungen, mit denen die beiden Ärztinnen und 14 Ärzte der LNG heute befasst sind. Täglich stehen 2 LNA rund um die Uhr auf Anforderung der FEZ bereit. Damit ist die Leitende Notarztgruppe nicht nur für die kommende Weltmeisterschaft gut gerüstet.

Zur Feier des 20-jährigen Bestehens kommt es im Rahmen des 3. Internationalen Emergency and Rescue Congress Intercon am 8. September um 19.00 Uhr zu einem Empfang im $\mathrm{CCH}$.

Dr. Michael Langhorst LNA Feuerwehr HH 